F. Reprod. Fert. (1970) 22, 469-482

\title{
SEPARATION OF RABBIT SEMEN INTO TWO POPULATIONS OF SPERMATOZOA BY CENTRIFUGATION
}

\author{
J. M. BRANHAM* \\ Institute of Animal Genetics, \\ West Mains Road, Edinburgh \\ (Received 29th Fuly 1969)
}

\begin{abstract}
Summary. Spermatozoa from semen centrifuged into Ficoll gradients separated into two populations. Under some conditions, they separated into flocculated and unflocculated populations. When flocculation was controlled, they separated into rapidly and slowly sedimenting populations. The centrifugal population consisted predominantly of active spermatozoa while the centripetal population contained predominantly inactive ones. Various morphologically distinct types of spermatozoa were also not randomly distributed. Experimentally inactivated, nonflocculating spermatozoa sedimented as a single population. It was concluded that flocculation and motility influenced sedimentation rate more markedly than other factors.
\end{abstract}

\section{INTRODUCTION}

Density and/or size differences between X- and Y-bearing spermatozoa have long been postulated (Lush, 1925; Harvey, 1946) and there have been numerous attempts to control genetic sex by separating spermatozoa by centrifugation. These attempts have occasionally been successful but, as yet, no dependable method for controlling sex by differential sedimentation has been perfected (cf. More O'Ferrall, Meacham \& Foreman, 1968; Bedford \& Bibeau, 1967). This difficulty perhaps results from a lack of information about factors influencing sperm sedimentation. Some of these factors were elucidated by the following experiments wherein rabbit semen was centrifuged into stabilized density gradient columns.

\section{MATERIALS AND METHODS}

Ejaculated semen was collected on a 2-day schedule (cf. Desjardins, Kirton \& Hafs, 1968) from Edinburgh AS strain bucks, known to be heterozygous at one locus, and allowed to cool to room temperature $\left(23 \pm 2^{\circ} \mathrm{C}\right)$ in about $15 \mathrm{~min}$.

Phosphate-buffered ( $\mathrm{pH} 7.6$ ) glucose-saline diluent (BM) was used (Beatty, 1964). Ficoll (Pharmacia, Lot 5990 or 8150 ) gradients were prepared by the double-mixing chamber technique, from two stock solutions, one of specific

\footnotetext{
* Present address: Department of Zoology, University of Hawaii, Honolulu.
} 
gravity 1.08 (cf. Beatty, 1964) and the other (a 1:1 dilution of the first with BM) of specific gravity of $1 \cdot 03$. The gradients were formed in glass centrifuge tubes and were $100 \pm 5 \mathrm{~mm}$ long and $13 \mathrm{~mm}$ in diameter. The centrifuge tubes were perforated at the bottom with a l-mm hole. This hole was plugged with Plasticine during the centrifugation and was easily opened for the controlled removal of the sample.

Semen was pre-treated as described below and layered 1 to $5 \mathrm{~mm}$ deep over the gradients. It was then centrifuged in an MSE Mistral L-2 centrifuge around a 22-mm maximum radius. Centrifugation was usually at $1000 \mathrm{rev} / \mathrm{min}$ for 30 $\min$ at $20^{\circ} \mathrm{C}$. After centrifugation, the tubes were photographed against a millimetre scale, with oblique lighting, and the sample removed through the bottom of the tube for further analysis.

\section{RESULTS}

When semen, freed of large bits of coagulum but otherwise unaltered, was centrifuged into density gradients, spermatozoa and other granular materials (cf. Metz, Hinsch \& Anika, 1968) sedimented as a broad band, with extensive flocculation at its front (Pl. 1, Fig. 1A). Clearly, separation depended on controlling this flocculation.

Two methods were devised to suppress flocculation (Pl. 1, Fig. 1). One (cf. Walton, 1924) involved altering the $\mathrm{pH}$ of the medium. This was accomplished by adding $0.2 \mathrm{~N}-\mathrm{NaOH}$ or $\mathrm{HCl}$ to the Ficoll solutions to produce the desired $\mathrm{pH}$. Flocculation decreased with increased $\mathrm{pH}$, to $\mathrm{pH} 9 \cdot 5$, above which tail-to-tail aggregates were observed (Bedford, 1965). Rabbit spermatozoa are surprisingly tolerant of high $\mathrm{pH}$ (Emmens, 1948).

The second method was suggested by the observation of Dott \& Walton (1960) that flocculates were dispersed by the nigrosin-eosin staining technique. Nigrosin was prepared as for nigrosin-eosin staining by dissolving $10 \%$ nigrosin (Gurr) in distilled water, refluxing for $8 \mathrm{hr}$ and filtering (Hancock, 1952). The $\mathrm{pH}$ (initially about 10 ) was adjusted to 7.6 with $\mathrm{HCl}$. Four or five drops of this solution were added to the semen samples (about $0.7 \mathrm{ml}$ ) before layering them over the gradients. Such treatment virtually prevented subsequent flocculation (PI. 1, Fig. 1F) without perceptibly altering sperm activity. Eosin alone had no effect or resulted in increased flocculation.

When flocculation was suppressed by either of these methods, spermatozoa

\section{EXPLANATION OF PLATE 1}

Fig. 1. Photographs of tubes showing flocculation of spermatozoa, at different levels of $\mathrm{pH}$ and pre-treated with nigrosin. Aliquots of semen $(0.2 \mathrm{ml})$ were centrifuged into dextran gradients adjusted to; (A) $\mathrm{pH} 7 \cdot 6$, (B) $\mathrm{pH} 8 \cdot 0$, (C) $\mathrm{pH} 8 \cdot 5$, (D) $\mathrm{pH} 9 \cdot 0$, (E) $\mathrm{pH}$ $9 \cdot 5,(\mathrm{~F}) \mathrm{pH} 7 \cdot 6$. The semen in $(\mathrm{F})$ was mixed with an equal volume of $10 \%$ nigrosin before overlayering.

FIg. 2. Distribution of washed spermatozoa after centrifugation. A sample of washed spermatozoa was divided and one half inactivated by heating to $58.5^{\circ} \mathrm{C}$ for $5 \mathrm{~min}$. Both samples were then mixed with four drops of $10 \%$ nigrosin and centrifuged into dextran gradients. The active sample (right) was withdrawn and the concentration (solid line, lower scale) and percentage of active spermatozoa (dashed line, upper scale) plotted against distance sedimented, in $\mathrm{mm}$ on the scale photographed against the tubes. 
PLATE 1
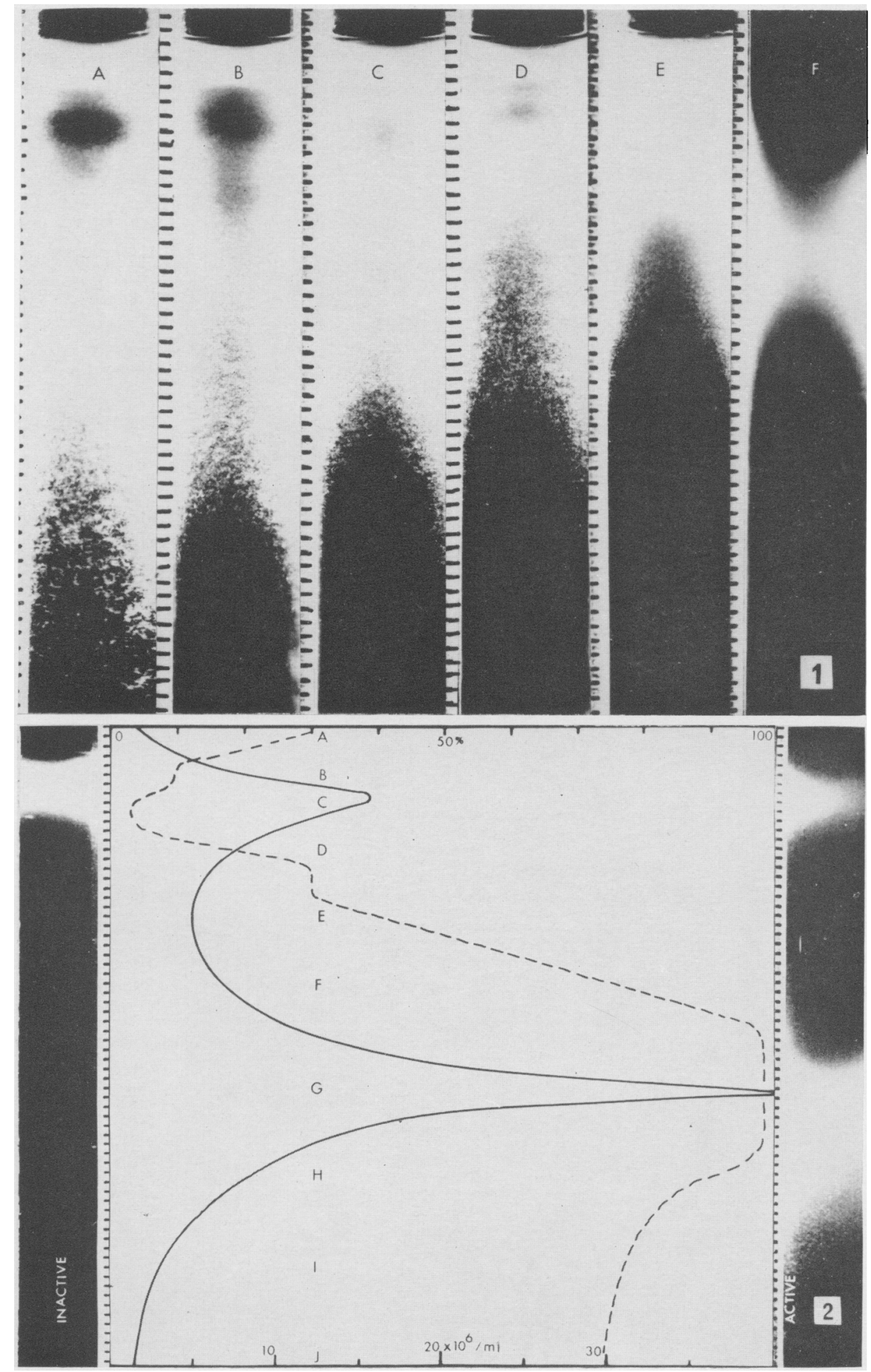

(Facing p. 470) 
PL.IIE ?

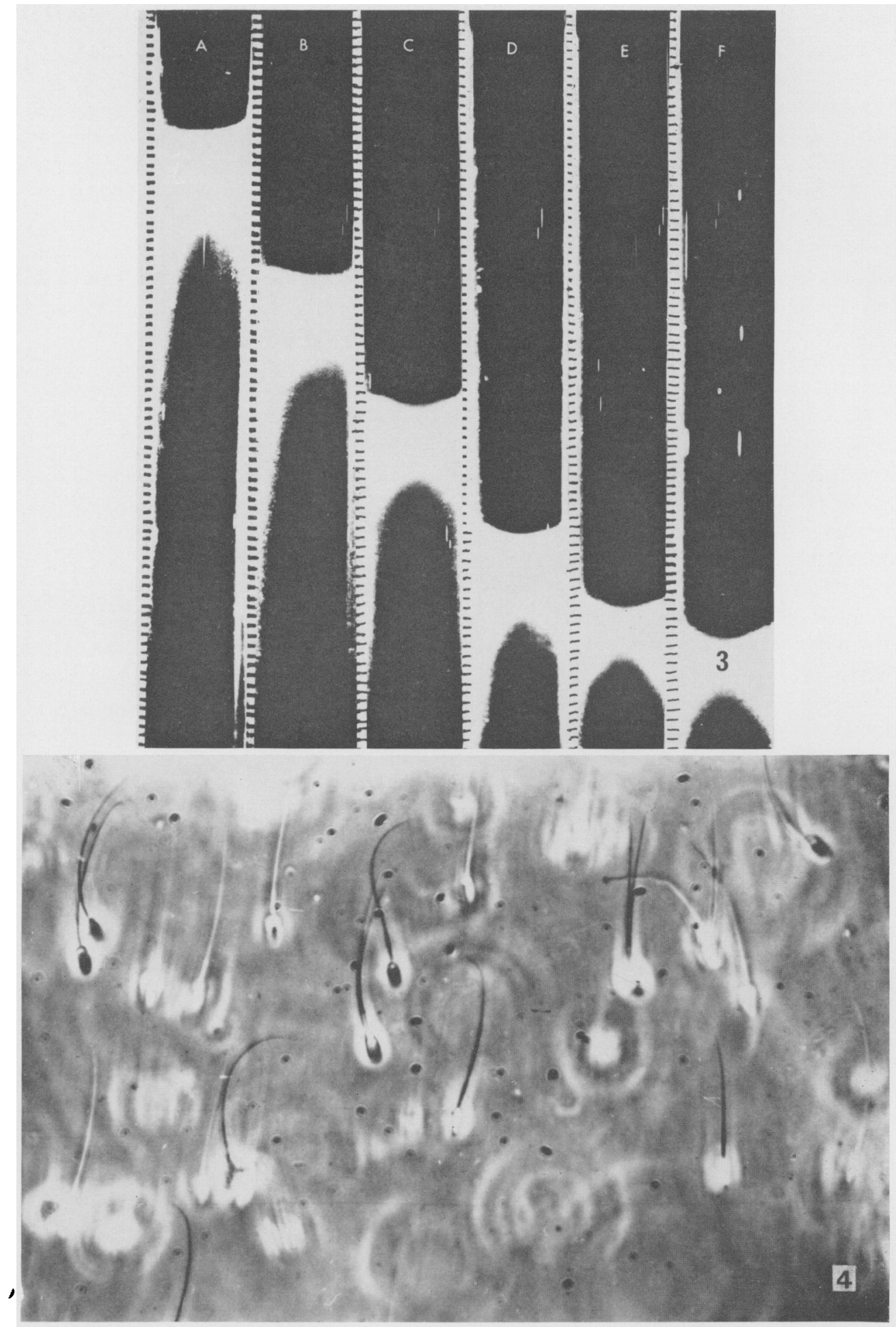

(Facing f). 471 ) 
and particulate material sedimented as a single broad band, with the particulate material concentrated at the front, followed by a dense population of active spermatozoa. These active spermatozoa were relatively free of granular materials. They were selectively removed from the tube, re-suspended in 10 volumes of $\mathrm{BM}$ and concentrated by centrifugation for $20 \mathrm{~min}$ at $1000 \mathrm{rev} / \mathrm{min}$ and are, hereafter, referred to as 'washed spermatozoa'.

Washed spermatozoa mixed with nigrosin, layered over Ficoll gradients and centrifuged as described above, sedimented as two distinct bands. Such centrifuged samples were removed in one-drop portions and the concentration of spermatozoa in each drop determined by haemocytometer count. Motility was sufficiently retarded by the high viscosity of the medium to permit direct counting of living spermatozoa. The proportion of 'active' spermatozoa in each drop was also noted ('activity' defined as any autonomous movement). The results of one experiment (rabbit 4lM) are presented in Pl. 1, Fig. 2.

It seemed from these results that differences in activity accounted for the observed separation into two populations. To test this, a sample of washed spermatozoa was divided into halves and one half was inactivated by heating to $58.5^{\circ} \mathrm{C}$ for $5 \mathrm{~min}$. (The conditions for inactivating spermatozoa were critical. All other attempts resulted in extensive flocculation upon sedimentation.) The active half was layered over one gradient and the inactivated half over another and both were centrifuged. The inactivated spermatozoa sedimented as a single band at the same rate as the slowly sedimenting band from the active half (see Pl. 1, Fig. 2). This experiment was repeated six times. In three of these determinations, the tube with the inactivated sample was photographed and returned to the centrifuge. Centrifugation was continued for $12 \mathrm{hr}$, with periodic stops to photograph the tubes. The inactivated spermatozoa remained in a single band as they sedimented to within $1.5 \mathrm{~cm}$ of the end of the tube (Pl. 2, Fig. 3).

A possible explanation of these observations was that active spermatozoa were orientated and swam centrifugally faster than inactive ones sedimented (Branham, 1966, 1969). To test for such orientation, spermatozoa were 'frozen' into gelatin during centrifugation. Gradients of 4 to $8 \%$ gelatin- $\mathrm{BM}(\mathrm{pH} \mathrm{7.6)}$ were formed at $35^{\circ} \mathrm{C}$. Semen was stirred into the upper two-thirds without upsetting the gradients and then centrifuged at $1000 \mathrm{rev} / \mathrm{min}$ at room temperature. When the centrifuge had reached speed, the refrigeration unit was turned on and set for $10^{\circ} \mathrm{C}$. Control tubes (uncentrifuged) were immersed in a beaker of ice water when the centrifuge began to chill. A second control was maintained at $35^{\circ} \mathrm{C}$ and drops observed periodically under the microscope to determine sperm activity. The gelled columns were removed from the centrifuge tube and sliced with a taut piano wire knife into slabs 1 to $2 \mathrm{~mm}$ thick.

\section{EXPLANATION OF PLATE 2}

Fig. 3. Sedimentation of heat-iriactivated spermatozoa after (A) $30 \mathrm{~min},(B) 60 \mathrm{~min},(\mathrm{C})$ $90 \mathrm{~min}$, (D) $130 \mathrm{~min}$, (E) $180 \mathrm{~min}$, (F) $12 \mathrm{hr}$.

Fig. 4. Spermatozoa trapped by gelation of the medium while being centrifuged at 1000 $\mathrm{re} / \mathrm{min}$. 
These slabs were examined and the spermatozoa within them were photographed.

Spermatozoa remained active for several hours in the gelatin medium at $35^{\circ} \mathrm{C}$. Their movements were, however, greatly retarded by the high viscosity.

After centrifugation, some spermatozoa were distributed into narrow periodic bands 1 to $10 \mathrm{~mm}$ apart. This banding could have resulted from saltatory progression of gelation upwards from the bottom of the tube. Spermatozoa in these bands were matted together and disorientated. Spermatozoa between the bands were mostly orientated in one direction (Pl. 2, Fig. 4). The direction of orientation, in some experiments, varied in different regions of the tube. In

TABLE 1

REGOGNIZED CHARACTERISTICS OF SPERMATOZOA AFTER NIGROSIN-EOSIN STAINING

Head Types

H1 Standard-The form usually represented (cf. Mann, 1964, p. 20)

H2 Distorted acrosomal cap; reduced to an anterior globule or with a distinctive anterior pointed protuberance

H3 Loose or missing acrosomal cap (cf. Mann, 1964, p. 24)

H4 Vacuolated nucleus

H5 Loose or missing post-nuclear cap

H6 Pyriform head (cf. Freund, 1966)

Neck Types

N1 Standard

N2 Swollen neck, exceeding the mid-piece in diameter

N3 Displaced, off-centre, attachment, usually accompanied by an off-centre cusp on the opposite side N4 Broken neck

Mid-piece Types

M1 Standard

M2 Expanded and loosened mid-piece

M3 Distal protoplasmic droplet (cf. Mann, 1964, p. 18)

M4 Proximal protoplasmic droplet

M5 Bent mid-piece

M6 Broken mid-piece

Tail Types

T1 Standard

T2 Two tails (perhaps, rouleaux of two spermatozoa)

T3 Broken tail

T4 Coiled tail (cf. Drevius \& Eriksson, 1966)

T5 Sharply bent tail

two experiments, spermatozoa from all regions examined in between bands were predominantly $(953 / 1000$ and $976 / 1000)$ orientated with their heads towards the centrifugal end of the tube. Uncentrifuged control tubes had no banding and the spermatozoa were randomly orientated.

The relationship between sedimentation rate and morphological characteristics of the spermatozoa was examined. Five semen samples were centrifuged into Ficoll gradients. The samples were withdrawn from the bottom of the tube in one-drop portions and sperm concentration and activity determined in selected drops. Another twenty to thirty selected drops were prepared for cytological examination by adding one drop of nigrosin-eosin to each and, after 
2 min, smearing the mixture on chromic acid-cleaned microslides. When the smears were dried, a cover slip was applied with Permount ${ }^{*}$.

Each slide was coded and examined by an impartial observer. In one experi-

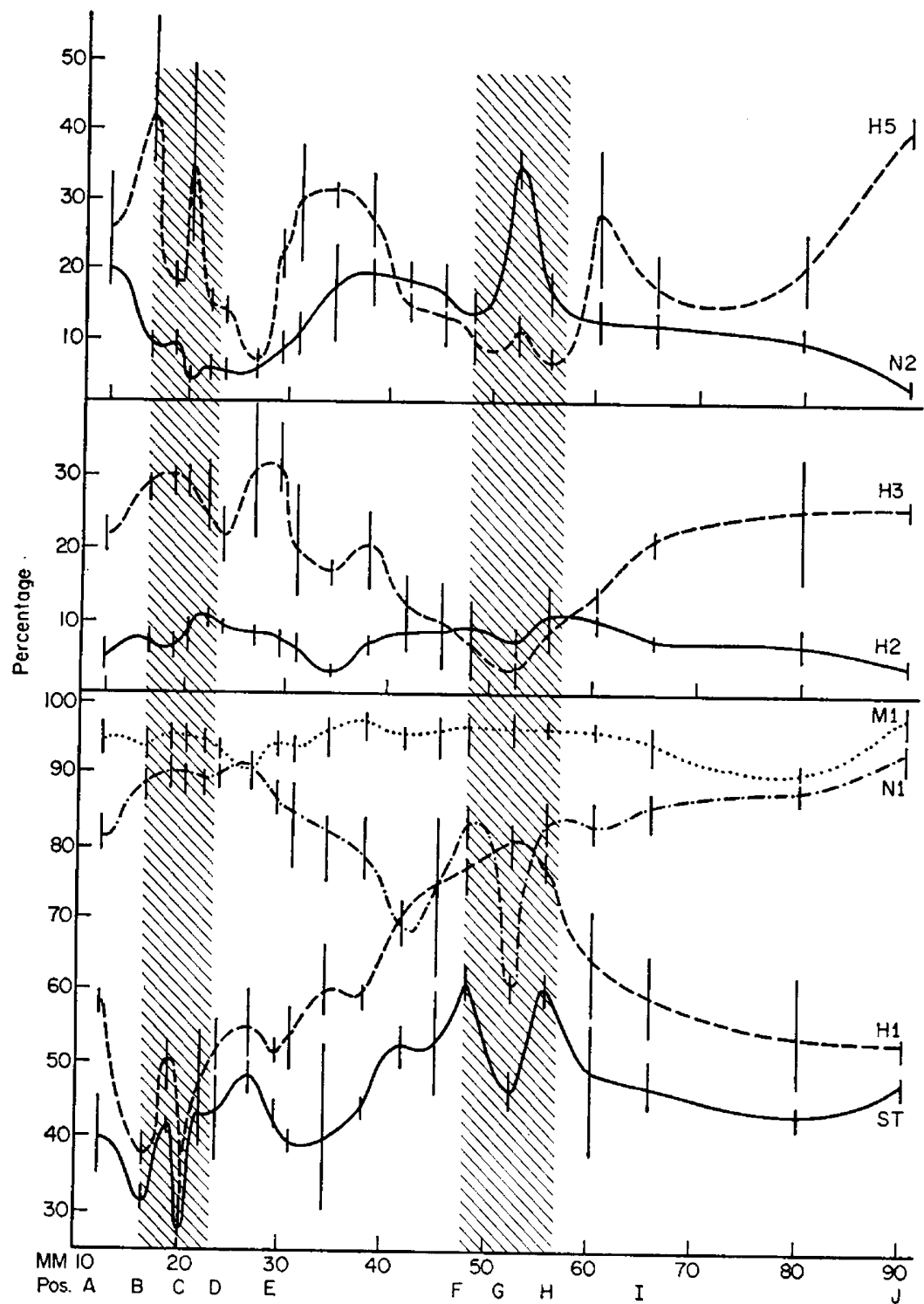

TExT-FIG. 1. The percentage of various recognizable types of spermatozoa plotted against the distance sedimented during centrifugation. The semen sample is the same as that pictured in Pl. I, Fig. 2 from which the positions (designated by letters) can be deter. mined for comparison with Table 2. The positions of the two dense populations is indicated by hatching. The types of spermatozoa are described in Table 1. Each slide was examined three times and averages and standard errors were calculated.

ment, the slides were re-coded and re-examined a total of three times, although the observer did not know that she was re-examining previously scored slides.

One hundred spermatozoa were scored on each slide. Each spermatozoon 
was compared with an array of photographs of distinctive sperm types arranged into categories. Six head-, four neck-, six mid-piece- and five tail-types were recognized and assigned numbers (Table 1). Each spermatozoon was scored by examining each part in turn, deciding which category (or categories) the part in question most closely resembled and recording the number representing that category. Thus, each spermatozoon was typed with at least four numbers (unless some part was absent).

In the five experiments, the concentration of spermatozoa and the percentage with various characteristics was determined at a number of locations in the column. The characteristics analysed included activity, the various morphological types and whether or not the head, neck, mid-piece and tail were all of the standard types. Eosin staining proved to be too difficult to judge under the circumstances and some of the morphological characteristics were too scarce for further analysis. The distributions of selected characteristics for one experiment are plotted in Text-fig. 1. The experiment plotted is the same as that depicted in Pl. 1, Fig. 2 and is the one in which each slide was scored a total of three times (from which standard errors were calculated). The characteristics not plotted were very sparsely represented in this sample.

The five experiments were not directly comparable. The various semen samples differed initially in the relative proportions of spermatozoa with the various characteristics analysed. Each sample separated into two populations but the distance sedimented and the relative proportion of spermatozoa in each band varied between samples. The experiments were compared by choosing

TABLE 2

COMPARISON BETWEEN FIVE EXPERIMENTS* OF THE PROPORTION OF SPERMATOZOA WITH VARIOUS CHARACTERISTICS $\dagger$ AT EQUIVALENT POSITIONS $\ddagger$ AFTER SEDIMENTATION

\begin{tabular}{|c|c|c|c|c|c|c|c|c|c|c|c|}
\hline Exp.‡ & $A$ & $B$ & $C$ & $D$ & $\underset{E}{P o}$ & $n^{*}{ }_{F}$ & $G$ & $H$ & $I$ & $\mathcal{F}$ & Control \\
\hline \multicolumn{12}{|c|}{$\%$ active } \\
\hline $\begin{array}{l}1 \\
3 \\
4 \\
5\end{array}$ & $\begin{array}{l}30 \\
52 \\
14 \\
10\end{array}$ & $\begin{array}{l}40 \\
22 \\
10 \\
10\end{array}$ & $\begin{array}{r}3 \\
14 \\
4 \\
10\end{array}$ & $\begin{array}{r}7 \\
4 \\
3 \\
10\end{array}$ & $\begin{array}{l}47 \\
68 \\
50 \\
20\end{array}$ & $\begin{array}{l}98 \\
92 \\
78 \\
60\end{array}$ & $\begin{array}{l}98 \\
98 \\
90 \\
86\end{array}$ & $\begin{array}{l}98 \\
94 \\
94 \\
96\end{array}$ & $\begin{array}{l}80 \\
87 \\
96 \\
82\end{array}$ & $\begin{array}{l}73 \\
92 \\
72 \\
70\end{array}$ & 90 \\
\hline
\end{tabular}

\begin{tabular}{lccccccccccc}
\multicolumn{10}{l}{ Standard head, neck, mid-piece and tail } \\
1 & 40 & 26 & 22 & 42 & 38 & 62 & 45 & 61 & 47 & 49 & 41 \\
& $\pm 6 \cdot 9$ & $\pm 1 \cdot 2$ & $\pm 6 \cdot 1$ & $\pm 8 \cdot 1$ & $\pm 11 \cdot 0$ & $\pm 11 \cdot 4$ & $\pm 5 \cdot 6$ & $\pm 1 \cdot 0$ & $\pm 4 \cdot 4$ & $\pm 4 \cdot 0$ & $\pm 6 \cdot 6$ \\
2 & 33 & 37 & 37 & 40 & 39 & 59 & 53 & 63 & 56 & 56 & 37 \\
3 & 38 & 41 & 38 & 41 & 53 & 73 & 46 & 39 & 71 & 75 & 53 \\
4 & 10 & 13 & 14 & 26 & 13 & 23 & 34 & 30 & 62 & 22 & 39 \\
5 & 46 & 40 & 40 & 35 & 35 & 47 & 58 & 50 & 73 & 62 & 57 \\
\hline
\end{tabular}

\begin{tabular}{lccccccccccc}
\hline \hline \multicolumn{1}{l}{ H1, Standard head } & & & & & & & & & \\
1 & $59 \cdot 3$ & $36 \cdot 3$ & $26 \cdot 0$ & $51 \cdot 0$ & $62 \cdot 0$ & $73 \cdot 0$ & $82 \cdot 0$ & $77 \cdot 0$ & $60 \cdot 3$ & $54 \cdot 3$ & $63 \cdot 0$ \\
& $\pm 1 \cdot 2$ & $\pm 4 \cdot 0$ & $\pm 4 \cdot 4$ & $\pm 6 \cdot 9$ & $\pm 6 \cdot 1$ & $\pm 3 \cdot 5$ & $\pm 4 \cdot 0$ & $\pm 2 \cdot 6$ & $\pm 6 \cdot 0$ & $\pm 4 \cdot 2$ & $\pm 13 \cdot 1$ \\
2 & 47 & 53 & 54 & 58 & 68 & 71 & 77 & 79 & 77 & 71 & 58 \\
3 & 65 & 64 & 61 & 60 & 63 & 87 & 60 & 54 & 80 & 85 & 87 \\
4 & 26 & 20 & 16 & 48 & 25 & 39 & 36 & 35 & 63 & 30 & 45 \\
5 & 55 & 53 & 45 & 51 & 47 & 58 & 76 & 69 & 77 & 68 & 68 \\
\hline
\end{tabular}




\begin{tabular}{|c|c|c|c|c|c|c|c|c|c|c|c|}
\hline $\operatorname{cxp} . \pm$ & $A$ & $B$ & $C$ & $D$ & & ${ }^{*}{ }_{F}^{*}$ & $G$ & $\boldsymbol{H}$ & $I$ & $\boldsymbol{J}$ & Control \\
\hline \multicolumn{12}{|c|}{$\mathrm{H} 2$, Globular or peaked acrosome } \\
\hline $\begin{array}{l}1 \\
2 \\
3 \\
4 \\
5\end{array}$ & $\begin{array}{r}6 \cdot 0 \\
+2 \cdot 6 \\
9 \\
1 \\
34 \\
11\end{array}$ & $\begin{array}{c}6 \cdot 3 \\
\pm 2 \cdot 1 \\
8 \\
5 \\
35 \\
11\end{array}$ & $\begin{array}{c}5 \cdot 0 \\
+0 \cdot 0 \\
6 \\
4 \\
11 \\
12\end{array}$ & $\begin{array}{c}10 \cdot 7 \\
\pm 1 \cdot 5 \\
9 \\
3 \\
36 \\
10\end{array}$ & $\begin{array}{c}3 \cdot 3 \\
\pm 0 \cdot 6 \\
8 \\
0 \\
24 \\
15\end{array}$ & $\begin{array}{c}7 \cdot 0 \\
\pm 1 \cdot 7 \\
30 \\
1 \\
48 \\
9\end{array}$ & $\begin{array}{r}6 \cdot 0 \\
+2 \cdot 6 \\
9 \\
4 \\
42 \\
16\end{array}$ & $\begin{array}{c}10 \cdot 3 \\
\pm 4 \cdot 2 \\
6 \\
3 \\
48 \\
11\end{array}$ & $\begin{array}{c}7 \cdot 0 \\
\pm 1 \cdot 0 \\
9 \\
0 \\
36 \\
9\end{array}$ & $\begin{array}{c}4 \cdot 3 \\
\pm 0 \cdot 6 \\
4 \\
2 \\
38 \\
14\end{array}$ & $\begin{array}{r}8 \cdot 3 \\
\pm 4 \cdot 5 \\
7 \\
3 \\
34 \\
12\end{array}$ \\
\hline \multicolumn{12}{|c|}{ H3, No acrosome } \\
\hline $\begin{array}{l}1 \\
2 \\
3 \\
4 \\
5\end{array}$ & $\begin{array}{l}20 \cdot 7 \\
\pm 4 \cdot 2 \\
39 \\
20 \\
21 \\
30\end{array}$ & $\begin{array}{c}28 \cdot 3 \\
\pm 4 \cdot 0 \\
32 \\
12 \\
26 \\
30\end{array}$ & $\begin{array}{l}26 \cdot 3 \\
\pm 5 \cdot 6 \\
26 \\
18 \\
26 \\
41\end{array}$ & $\begin{array}{l}23 \cdot 0 \\
\pm 7 \cdot 8 \\
27 \\
27 \\
40 \\
37\end{array}$ & $\begin{array}{c}15 \cdot 7 \\
\pm 2 \cdot 5 \\
21 \\
22 \\
47 \\
35\end{array}$ & $\begin{array}{r}5 \cdot 7 \\
\pm 2 \cdot 1 \\
2 \\
1 \\
5 \\
30\end{array}$ & $\begin{array}{c}3 \cdot 0 \\
\pm 1 \cdot 7 \\
11 \\
16 \\
19 \\
11\end{array}$ & $\begin{array}{c}11 \cdot 0 \\
\pm 1 \cdot 7 \\
3 \\
14 \\
13 \\
22\end{array}$ & $\begin{array}{c}21 \cdot 0 \\
\pm 2 \cdot 0 \\
9 \\
7 \\
1 \\
8\end{array}$ & $\begin{array}{c}23 \cdot 7 \\
\pm 3 \cdot 1 \\
8 \\
5 \\
22 \\
12\end{array}$ & $\begin{array}{c}15 \cdot 7 \\
\pm 5 \cdot 0 \\
20 \\
7 \\
20 \\
18\end{array}$ \\
\hline \multicolumn{12}{|c|}{ H4, Vacuolated head } \\
\hline $\begin{array}{l}1 \\
2 \\
3 \\
4 \\
5\end{array}$ & $\begin{array}{c}1 \cdot 0 \\
\pm 1 \cdot 1 \\
0 \\
1 \\
2 \\
1\end{array}$ & $\begin{array}{c}0.0 \\
\pm 1 \cdot 1 \\
2 \\
1 \\
4 \\
2\end{array}$ & $\begin{array}{c}3 \cdot 0 \\
\pm 2 \cdot 6 \\
2 \\
1 \\
1 \\
3\end{array}$ & $\begin{array}{c}2 \cdot 3 \\
\pm 2 \cdot 1 \\
1 \\
1 \\
2 \\
2\end{array}$ & $\begin{array}{c}0.3 \\
+0.6 \\
0 \\
5 \\
4 \\
1\end{array}$ & $\begin{array}{c}1.0 \\
\pm 1.0 \\
2 \\
0 \\
2 \\
0\end{array}$ & $\begin{array}{c}1.3 \\
\pm 0.6 \\
0 \\
0 \\
1 \\
3\end{array}$ & $\begin{array}{c}0.0 \\
\pm 0.0 \\
0 \\
1 \\
2 \\
9\end{array}$ & $\begin{array}{c}0.7 \\
\pm 1.2 \\
0 \\
1 \\
0 \\
2\end{array}$ & $\begin{array}{c}0 \cdot 0 \\
\pm 0 \cdot 0 \\
6 \\
1 \\
4 \\
2\end{array}$ & $\begin{array}{c}9 \cdot 0 \\
\pm 6 \cdot 1 \\
8 \\
1 \\
0 \\
0\end{array}$ \\
\hline \multicolumn{12}{|c|}{ H5, Loosened or missing post-nuclear cap } \\
\hline $\begin{array}{l}1 \\
2 \\
3 \\
4 \\
5\end{array}$ & $\begin{array}{c}23 \cdot 3 \\
\pm 7 \cdot 8 \\
7 \\
29 \\
28 \\
1\end{array}$ & $\begin{array}{c}46.0 \\
\pm 8.5 \\
5 \\
22 \\
46 \\
9\end{array}$ & $\begin{array}{c}18 \cdot 7 \\
\pm 6 \cdot 1 \\
14 \\
15 \\
26 \\
1\end{array}$ & $\begin{array}{c}23 \cdot 7 \\
+13 \cdot 6 \\
7 \\
25 \\
49 \\
2\end{array}$ & $\begin{array}{c}27 \cdot 3 \\
\pm 5 \cdot 7 \\
2 \\
22 \\
5 \\
4\end{array}$ & $\begin{array}{c}12 \cdot 3 \\
\pm 3 \cdot 1 \\
3 \\
33 \\
12 \\
5\end{array}$ & $\begin{array}{c}10 \cdot 7 \\
\pm 3 \cdot 1 \\
9 \\
32 \\
4 \\
7\end{array}$ & $\begin{array}{c}5 \cdot 7 \\
+2 \cdot 5 \\
16 \\
42 \\
1 \\
11\end{array}$ & $\begin{array}{c}28.7 \\
\pm 9 \cdot 2 \\
7 \\
18 \\
0 \\
6\end{array}$ & $\begin{array}{c}40 \cdot 0 \\
\pm 3 \cdot 0 \\
14 \\
8 \\
14 \\
4\end{array}$ & $\begin{array}{c}9 \cdot 0 \\
\pm 1 \cdot 7 \\
4 \\
2 \\
1 \\
0\end{array}$ \\
\hline \multicolumn{12}{|c|}{ N1, Standard neck } \\
\hline $\begin{array}{l}1 \\
2 \\
3 \\
4 \\
5\end{array}$ & $\begin{array}{c}80 \cdot 0 \\
\pm 5 \cdot 6 \\
93 \\
83 \\
93 \\
95\end{array}$ & $\begin{array}{c}92 \cdot 0 \\
\pm 2 \cdot 0 \\
83 \\
90 \\
90 \\
85\end{array}$ & $\begin{array}{c}96.0 \\
\pm 2 \cdot 0 \\
91 \\
89 \\
95 \\
91\end{array}$ & $\begin{array}{c}92 \cdot 0 \\
\pm 5 \cdot 3 \\
88 \\
81 \\
93 \\
80\end{array}$ & $\begin{array}{c}79 \cdot 0 \\
\pm 5 \cdot 6 \\
77 \\
76 \\
62 \\
85\end{array}$ & $\begin{array}{c}77 \cdot 7 \\
\pm 5 \cdot 5 \\
93 \\
88 \\
87 \\
85\end{array}$ & $\begin{array}{c}61 \cdot 7 \\
\pm 3 \cdot 1 \\
76 \\
86 \\
93 \\
84\end{array}$ & $\begin{array}{c}84 \cdot 7 \\
\pm 4 \cdot 0 \\
90 \\
88 \\
94 \\
88\end{array}$ & $\begin{array}{c}85 \cdot 7 \\
\pm 5 \cdot 0 \\
76 \\
91 \\
99 \\
96\end{array}$ & $\begin{array}{c}93.3 \\
\pm 1.5 \\
84 \\
92 \\
92 \\
91\end{array}$ & $\begin{array}{l}70 \cdot 0 \\
\pm 6 \cdot 9 \\
73 \\
67 \\
97 \\
95\end{array}$ \\
\hline \multicolumn{12}{|c|}{ N2, Swollen neck } \\
\hline $\begin{array}{l}1 \\
2 \\
3 \\
4 \\
5 \\
5\end{array}$ & $\begin{array}{c}17 \cdot 0 \\
+4 \cdot 4 \\
4 \\
15 \\
4 \\
3\end{array}$ & $\begin{array}{c}6 \cdot 7 \\
\pm 2 \cdot 1 \\
12 \\
7 \\
6 \\
18\end{array}$ & $\begin{array}{c}3 \cdot 0 \\
\pm 1 \cdot 0 \\
5 \\
7 \\
4 \\
5\end{array}$ & $\begin{array}{c}4 \cdot 3 \\
\pm 2 \cdot 3 \\
12 \\
16 \\
14 \\
7\end{array}$ & $\begin{array}{c}16 \cdot 0 \\
\pm 4 \cdot 4 \\
18 \\
4 \\
3 \\
7\end{array}$ & $\begin{array}{c}13 \cdot 0 \\
\pm 3 \cdot 0 \\
6 \\
13 \\
11 \\
11\end{array}$ & $\begin{array}{c}33 \cdot 3 \\
\pm 2 \cdot 5 \\
18 \\
14 \\
5 \\
10\end{array}$ & $\begin{array}{c}13.7 \\
\pm 4 \cdot 0 \\
9 \\
11 \\
5 \\
15\end{array}$ & $\begin{array}{c}11 \cdot 0 \\
+3.6 \\
21 \\
7 \\
1 \\
3\end{array}$ & $\begin{array}{c}4 \cdot 7 \\
+2 \cdot 5 \\
10 \\
7 \\
8 \\
9\end{array}$ & $\begin{array}{c}26 \cdot 7 \\
\pm 9 \cdot 2 \\
20 \\
30 \\
2 \\
1\end{array}$ \\
\hline \multicolumn{12}{|c|}{ N3, Off-centre neck with a post-nuclear cusp } \\
\hline 1 & $\begin{array}{c}2 \cdot 0 \\
+1 \cdot 7 \\
2 \\
0 \\
1 \\
1\end{array}$ & $\begin{array}{c}1 \cdot 0 \\
\pm 1 \cdot 0 \\
5 \\
1 \\
2 \\
0\end{array}$ & $\begin{array}{c}0 \cdot 7 \\
\pm 1 \cdot 2 \\
3 \\
0 \\
4 \\
0\end{array}$ & $\begin{array}{c}2 \cdot 3 \\
+0 \cdot 6 \\
2 \\
1 \\
2 \\
0\end{array}$ & $\begin{array}{c}4 \cdot 0 \\
+2 \cdot 6 \\
1 \\
0 \\
0 \\
0\end{array}$ & $\begin{array}{c}1 \cdot 7 \\
\pm 1 \cdot 5 \\
1 \\
0 \\
1 \\
0\end{array}$ & $\begin{array}{c}5 \cdot 0 \\
\pm 1 \cdot 0 \\
6 \\
0 \\
0 \\
1\end{array}$ & $\begin{array}{c}1 \cdot 7 \\
\pm 1 \cdot 5 \\
1 \\
1 \\
1 \\
1\end{array}$ & $\begin{aligned} & 3 \cdot 0 \\
& \pm 0 \cdot 0 \\
& 3 \\
& 2 \\
& 0 \\
& 0\end{aligned}$ & $\begin{array}{c}0 \cdot 3 \\
\pm 0.6 \\
6 \\
0 \\
0 \\
0\end{array}$ & $\begin{array}{c}3 \cdot 0 \\
\pm 1 \cdot 7 \\
2 \\
0 \\
0 \\
1\end{array}$ \\
\hline \multicolumn{12}{|c|}{ N4, Broken neck } \\
\hline $\begin{array}{l}1 \\
2 \\
3 \\
4 \\
5\end{array}$ & $\begin{array}{c}0.7 \\
+1.2 \\
0 \\
9 \\
11 \\
0\end{array}$ & $\begin{array}{c}9 \cdot 0 \\
\pm 0 \cdot 0 \\
1 \\
1 \\
2 \\
2\end{array}$ & $\begin{array}{c}0 \cdot 3 \\
+0 \cdot 6 \\
1 \\
4 \\
2 \\
3\end{array}$ & $\begin{array}{c}0.0 \\
\pm 0.6 \\
2 \\
3 \\
2 \\
9\end{array}$ & $\begin{array}{c}2 \cdot 3 \\
\pm 0 \cdot 6 \\
4 \\
20 \\
33 \\
9\end{array}$ & $\begin{array}{c}0.3 \\
\pm 0.6 \\
0 \\
2 \\
1 \\
7\end{array}$ & $\begin{array}{c}0.3 \\
\pm 0.6 \\
0 \\
0 \\
2 \\
3\end{array}$ & $\begin{array}{c}0.0 \\
\pm 0.0 \\
0 \\
0 \\
0 \\
0\end{array}$ & $\begin{aligned} & 0.7 \\
& \pm 0.6 \\
& 0 \\
& 0 \\
& 0 \\
& 1\end{aligned}$ & $\begin{array}{c}1 \cdot 0 \\
\pm 1.7 \\
0 \\
2 \\
0 \\
0\end{array}$ & $\begin{array}{c}0 \cdot 3 \\
\pm 0 \cdot 6 \\
1 \\
3 \\
3 \\
2\end{array}$ \\
\hline
\end{tabular}




\begin{tabular}{|c|c|c|c|c|c|c|c|c|c|c|c|}
\hline & & & & & Posi & tion* & & & & & \\
\hline Exp. & $A$ & $B$ & $C$ & $D$ & $E$ & $F$ & $G$ & $H$ & $I$ & $\mathcal{F}$ & Control \\
\hline M1, & Standa & dd mid- & iece & & & & & & & & \\
\hline $\begin{array}{l}1 \\
2 \\
3 \\
4 \\
5 \\
\end{array}$ & $\begin{array}{c}96 \cdot 3 \\
\pm 3 \cdot 2 \\
85 \\
83 \\
82 \\
93\end{array}$ & $\begin{array}{c}94 \cdot 7 \\
\pm 4 \cdot 0 \\
89 \\
82 \\
94 \\
82\end{array}$ & $\begin{array}{l}96 \cdot 7 \\
\pm 0 \cdot 6 \\
81 \\
67 \\
74 \\
90 \\
\end{array}$ & $\begin{array}{l}90 \cdot 0 \\
\pm 2 \cdot 0 \\
82 \\
88 \\
90 \\
79\end{array}$ & $\begin{array}{c}94 \cdot 3 \\
\pm 2 \cdot 5 \\
88 \\
92 \\
73 \\
85\end{array}$ & $\begin{array}{c}97 \cdot 3 \\
\pm 3 \cdot 1 \\
97 \\
96 \\
80 \\
91\end{array}$ & $\begin{array}{c}96 \cdot 7 \\
\pm 2 \cdot 1 \\
92 \\
97 \\
90 \\
87\end{array}$ & $\begin{array}{c}96 \cdot 3 \\
\pm 1 \cdot 2 \\
100 \\
96 \\
92 \\
85 \\
\end{array}$ & $\begin{array}{c}96 \cdot 0 \\
\pm 2 \cdot 0 \\
100 \\
96 \\
96 \\
98\end{array}$ & $\begin{array}{c}97 \cdot 3 \\
\pm 2 \cdot 3 \\
93 \\
95 \\
82 \\
98\end{array}$ & $\begin{array}{l}93 \cdot 7 \\
\pm 1.5 \\
92 \\
90 \\
87 \\
92 \\
\end{array}$ \\
\hline M2, & Loosen & ed mid- & iece & & & & & & & & \\
\hline $\begin{array}{l}1 \\
2 \\
3 \\
4 \\
5\end{array}$ & $\begin{array}{c}1 \cdot 0 \\
\pm 1 \cdot 7 \\
2 \\
6 \\
3 \\
1\end{array}$ & $\begin{array}{c}1 \cdot 7 \\
\pm 2 \cdot 1 \\
1 \\
2 \\
0 \\
7\end{array}$ & $\begin{array}{c}0.3 \\
\pm 0 \cdot 6 \\
0 \\
12 \\
4 \\
3\end{array}$ & $\begin{array}{c}3 \cdot 3 \\
\pm 2 \cdot 5 \\
5 \\
8 \\
0 \\
3\end{array}$ & $\begin{array}{c}1 \cdot 0 \\
\pm 1 \cdot 7 \\
4 \\
7 \\
3 \\
12\end{array}$ & $\begin{array}{c}1.0 \\
\pm 1.0 \\
1 \\
1 \\
1 \\
1\end{array}$ & $\begin{array}{c}0.0 \\
\pm 0.0 \\
1 \\
5 \\
1 \\
11\end{array}$ & $\begin{array}{c}0.3 \\
\pm 0 \cdot 6 \\
0 \\
1 \\
0 \\
6\end{array}$ & $\begin{array}{c}1 \cdot 7 \\
\pm 1 \cdot 5 \\
0 \\
2 \\
0 \\
1\end{array}$ & $\begin{array}{c}0.3 \\
\pm 0.6 \\
0 \\
3 \\
10 \\
2\end{array}$ & $\begin{array}{c}3 \cdot 7 \\
\pm 2 \cdot 1 \\
2 \\
3 \\
0 \\
0\end{array}$ \\
\hline M3, & Distal & orotopla & mic $\mathrm{dr}$ & plet & & & & & & & \\
\hline $\begin{array}{l}1 \\
2 \\
3 \\
4 \\
5\end{array}$ & $\begin{array}{c}1 \cdot 7 \\
\pm 1.5 \\
6 \\
3 \\
13 \\
3\end{array}$ & $\begin{array}{c}3 \cdot 3 \\
\pm 1.5 \\
6 \\
6 \\
19 \\
5\end{array}$ & $\begin{array}{c}1.3 \\
+0.6 \\
14 \\
5 \\
6 \\
3\end{array}$ & $\begin{array}{r}5 \cdot 7 \\
\pm 3 \cdot 5 \\
7 \\
6 \\
10 \\
4\end{array}$ & $\begin{array}{c}2 \cdot 7 \\
\pm 1.5 \\
5 \\
3 \\
17 \\
9\end{array}$ & $\begin{array}{r}3.7 \\
\pm 1.5 \\
3 \\
4 \\
16 \\
5\end{array}$ & $\begin{array}{c}2 \cdot 0 \\
+1.0 \\
5 \\
0 \\
8 \\
1\end{array}$ & $\begin{array}{c}2 \cdot 7 \\
+0 \cdot 6 \\
0 \\
3 \\
5 \\
2 \\
\end{array}$ & $\begin{array}{c}2 \cdot 0 \\
+2 \cdot 6 \\
0 \\
1 \\
3 \\
1\end{array}$ & $\begin{array}{c}1 \cdot 3 \\
\pm 1 \cdot 2 \\
3 \\
1 \\
8 \\
0\end{array}$ & $\begin{array}{c}1 \cdot 7 \\
+0 \cdot 6 \\
2 \\
2 \\
9 \\
1\end{array}$ \\
\hline M4 & Proxi & al pro & asm & rople & & & & & & & \\
\hline $\begin{array}{l}3 \\
4 \\
5\end{array}$ & $\begin{array}{c}0.3 \\
\pm 0.6 \\
3 \\
5 \\
1 \\
2\end{array}$ & $\begin{array}{c}0.3 \\
\pm 0.6 \\
1 \\
9 \\
1 \\
0\end{array}$ & $\begin{array}{c}1 \cdot 7 \\
\pm 0 \cdot 6 \\
3 \\
15 \\
0 \\
2\end{array}$ & $\begin{array}{c}2 \cdot 3 \\
\pm 1 \cdot 5 \\
5 \\
5 \\
1 \\
6\end{array}$ & $\begin{array}{c}0.3 \\
\pm 0.6 \\
3 \\
4 \\
1 \\
2\end{array}$ & $\begin{array}{c}1.0 \\
+1.7 \\
4 \\
2 \\
3 \\
1\end{array}$ & $\begin{array}{c}1 \cdot 0 \\
+0 \cdot 6 \\
4 \\
4 \\
1 \\
7\end{array}$ & $\begin{array}{c}0 \cdot 7 \\
+1 \cdot 2 \\
0 \\
1 \\
3 \\
6\end{array}$ & $\begin{array}{c}1 \cdot 0 \\
\pm 1 \cdot 0 \\
0 \\
1 \\
1 \\
0\end{array}$ & $\begin{array}{c}1 \cdot 0 \\
\pm 1 \cdot 0 \\
4 \\
1 \\
0 \\
0\end{array}$ & $\begin{array}{c}1 \cdot 0 \\
+1 \cdot 0 \\
2 \\
5 \\
2 \\
5\end{array}$ \\
\hline M5, & Bent $m$ & id-piece & & & & & & & & & \\
\hline $\begin{array}{l}1 \\
2 \\
3 \\
4 \\
5\end{array}$ & $\begin{array}{c}0 \cdot 7 \\
\pm 1 \cdot 2 \\
3 \\
4 \\
0 \\
2\end{array}$ & $\begin{array}{c}0.7 \\
\pm 0.6 \\
3 \\
3 \\
0 \\
7\end{array}$ & $\begin{array}{c}0.0 \\
\pm 0.0 \\
1 \\
2 \\
1 \\
3\end{array}$ & $\begin{array}{c}0.7 \\
\pm 1 \cdot 2 \\
4 \\
1 \\
0 \\
5\end{array}$ & $\begin{array}{c}1 \cdot 0 \\
\pm 1 \cdot 0 \\
2 \\
1 \\
4 \\
3\end{array}$ & $\begin{array}{c}0 \cdot 0 \\
\pm 0 \cdot 0 \\
2 \\
2 \\
0 \\
4\end{array}$ & $\begin{array}{c}0.3 \\
\pm 0.6 \\
1 \\
0 \\
1 \\
1\end{array}$ & $\begin{array}{c}0.0 \\
\pm 0 \cdot 0 \\
0 \\
1 \\
0 \\
1\end{array}$ & $\begin{array}{c}0.0 \\
\pm 0.0 \\
0 \\
0 \\
0 \\
0\end{array}$ & $\begin{array}{c}0.0 \\
\pm 0.0 \\
0 \\
0 \\
0 \\
0\end{array}$ & $\begin{array}{c}0.3 \\
\pm 0.6 \\
1 \\
0 \\
2 \\
2\end{array}$ \\
\hline Tail & , other & han sta & dard $t$ & & & & & & & & \\
\hline $\begin{array}{l}1 \\
2 \\
3 \\
4 \\
5\end{array}$ & $\begin{array}{c}1 \cdot 0 \\
\pm 1 \cdot 0 \\
0 \\
6 \\
3 \\
4\end{array}$ & $\begin{array}{c}1 \cdot 0 \\
\pm 1 \cdot 0 \\
3 \\
4 \\
2 \\
2\end{array}$ & $\begin{array}{c}0.3 \\
\pm 0.6 \\
1 \\
0 \\
0 \\
3\end{array}$ & $\begin{array}{c}1.7 \\
\pm 0.6 \\
1 \\
2 \\
0 \\
6\end{array}$ & $\begin{array}{c}1 \cdot 7 \\
\pm 1 \cdot 5 \\
2 \\
0 \\
2 \\
2\end{array}$ & $\begin{array}{c}0.3 \\
\pm 0.6 \\
0 \\
1 \\
1 \\
6\end{array}$ & $\begin{array}{c}0.7 \\
\pm 1.2 \\
3 \\
0 \\
1 \\
2\end{array}$ & $\begin{array}{c}0 \cdot 3 \\
+0 \cdot 6 \\
4 \\
0 \\
1 \\
6\end{array}$ & $\begin{array}{c}0.3 \\
\pm 0.6 \\
1 \\
1 \\
0 \\
0\end{array}$ & $\begin{array}{c}0.0 \\
\pm 0.0 \\
0 \\
1 \\
2 \\
1\end{array}$ & $\begin{array}{c}0.3 \\
\pm 0.6 \\
1 \\
3 \\
1 \\
0\end{array}$ \\
\hline
\end{tabular}

Rabbits used in different experiments:

* Exp. 1, Rabbit $41 \mathrm{M}$ (same as Text-fig. 1); Exp. 2, 41M; Exp. 3, 32P; Exp. 4, Z54; Exp. 5, 3V.

$\dagger$ See Table 1 for description of characteristics.

\$ See Pl. 1, Fig. 2 and Text-fig. 1 for relative position of the selected drops.

drops from equivalent parts of the distribution curves of each one. The locations of the drops relative to the sample shown in Text-fig. 1 are designated with letters in P1. 1, Fig. 2 and Text-fig. 1. The proportion of spermatozoa with the selected characteristics varied with the location in the columns in each experiment but not always in the same pattern, as can be seen from Table 2 .

During the preceding experiments, distinct differences between the sedimentation of various semen samples were observed. These differences were examined by simultaneously centrifuging and directly comparing six different 
semen samples. The procedure was repeated four times. In the first three of these comparisons, the number of spermatozoa varied between samples but in the fourth, this was standardized. The most obvious differences included the extent of flocculation, the relative concentration of spermatozoa in the two populations, the distances sedimented by the two populations (Table 3), and the spread of the faster of the two populations. Further analysis of these differences might prove useful in the evaluation of semen samples.

Differences between the gradients apparently did not account for the observed differences. This was demonstrated by preparing six gradients as above and layering equivalent portions of the same semen samples over each. All six samples looked alike after centrifugation.

Sperm concentration similarly had little effect on sedimentation rate. A sample of washed sperm was diluted in five two-fold steps and a portion of each

\section{TABLE 3}

COMPARISON BETWEEN DIFFERENT SEMEN SAMPLES, OF THE DISTANGES (IN MM) SEDIMENTED BY THE TWO POPULATIONS OF SPERMATOZOA IN FOUR SEPARATE DETERMINATIONS

\begin{tabular}{c|c|cccccc}
\hline & & \multicolumn{7}{|c}{ Semen from rabbit } \\
Exp. & Population & $Z 54$ & $41 M$ & $3 V$ & $36 P$ & $35 R$ & $32 P$ \\
\hline 1 & Centrifugal & 33 & 38 & 30 & 33 & 36 & 29 \\
& Centripetal & 12 & 12 & 13 & 13 & 14 & 14 \\
& Difference & 21 & 26 & 17 & 20 & 22 & 15 \\
\hline 2 & Centrifugal & 20 & 24 & 22 & 24 & 19 & 27 \\
& Centripetal & 12 & 13 & 11 & 13 & 12 & 12 \\
& Difference & 18 & 11 & 11 & 11 & 7 & 15 \\
\hline 3 & Centrifugal & 23 & 32 & - & 27 & 27 & 33 \\
& Centripetal & 11 & 12 & - & 14 & 14 & 13 \\
& Difference & 12 & 19 & - & 13 & 13 & 20 \\
\hline 4 & Centrifugal & 38 & 42 & 43 & 30 & 37 & 39 \\
& Centripetal & 12 & 12 & 16 & 14 & 15 & 14 \\
& Difference & 26 & 30 & 27 & 16 & 22 & 25 \\
& & & & & & & \\
\hline
\end{tabular}

dilution was layered over a separate gradient and centrifuged. The distances sedimented by the centre of concentration of both the 'rapid' and the 'slow' populations were the same at all five concentrations. Flocculation and the spread of the more rapidly sedimenting population also appeared about the same in all concentrations but the spread of the slower population increased with increased concentration.

The distance that the more rapid band sedimented and the extent of flocculation increased with increased hydrogen ion activity. In one experiment, equivalent portions of a washed (nigrosin-treated) semen sample were layered over gradients of $\mathrm{pH} 6 \cdot 0,7 \cdot 6$ and 9.0. The concentration peak of the centripetal population sedimented $11 \mathrm{~mm}$ in all three samples. The centrifugal population sedimented $54 \mathrm{~mm}$ at $\mathrm{pH} 6 \cdot 0,47 \mathrm{~mm}$ at $\mathrm{pH} 7 \cdot 6$ and $35 \mathrm{~mm}$ at $\mathrm{pH} 9 \cdot 0$. 
Flocculation was noticeable and about the same at $\mathrm{pH} 7.6$ and 6.0 but absent at $\mathrm{pH} 9 \cdot 0$. Similar results were obtained in two other experiments.

The fertility of spermatozoa after separation was also examined. A number of does destined to be discarded from the colony for various reasons (including age, 4 to 5 years, and inability to produce or rear offspring) were available for these tests. Such does are frequently able to produce blastocysts, as can be seen from the results, presented in Tables 4 to 6 , and therefore are economically useful for detecting fertilization. They were injected with 25 units of chorionic gonadotrophin (Pregnol ${ }^{\star}$ ) and inseminated as described by Adams (1962). Three days later they were killed to determine numbers of corpora lutea and blastocysts (flushed from the uteri).

TABLE 4

FERTILITY OF SEMEN AFTER HIGH PH AND NIGROSIN ANTI-FLOCCULATION TREATMENT

\begin{tabular}{l|c|c|c}
\hline & \multicolumn{3}{|c}{ Pre-treatment of semen } \\
\cline { 2 - 4 } & None & None & Nigrosin \\
\hline pH of gradient & $7 \cdot 6$ & $9 \cdot 5$ & $7 \cdot 6$ \\
Blastocysts & 40 & $\frac{16}{30}$ & $\frac{0}{32}$ \\
Corpora lutea & $\overline{90}$ & 53 & 0 \\
$\%$ Fertilization & 44 & 53 & \\
\hline
\end{tabular}

To test the effect of the flocculation-prevention treatment on fertility, semen samples were centrifuged once into gradients and the resultant 'clouds' of spermatozoa were inseminated. The results, presented in Table 4, indicated that nigrosin treatment rendered the spermatozoa infertile. This effect was reversed by 'washing' the spermatozoa and re-suspending them in seminal plasma before insemination. In this experiment (results in Table 5), semen samples were mixed with nigrosin and centrifuged into gradients $(\mathrm{pH} 7 \cdot 6)$. The clouds of spermatozoa were withdrawn and divided into three portions. The first portion was put directly into a centrifuge tube, the second and third were diluted with $10 \mathrm{vol}$ of $\mathrm{BM}$. The three tubes were then centrifuged at

TABLE 5

FERTILITY OF NIGROSIN-TREATED SPERMATOZOA AFTER WASHING AND RE-SUSPENSION IN SEMINAL PLASMA

\begin{tabular}{|c|c|c|c|c|}
\hline & \multirow{2}{*}{$\frac{\text { Control semen }}{\begin{array}{c}\text { Inseminated in } \\
8 \% \text { dextran }\end{array}}$} & \multicolumn{3}{|c|}{$\begin{array}{l}\text { Nigrasin-treated spermatozoa } \\
\text { recovered from dextran gradients }\end{array}$} \\
\hline & & $8 \%$ dextran & minat & $\begin{array}{l}\text { in: } \\
\text { seminal plasma }\end{array}$ \\
\hline$\frac{\text { Blastocysts }}{\text { Corpora lutea }}$ & $\frac{46}{60}$ & $\frac{0}{44}$ & $\frac{11}{32}$ & $\frac{31}{41}$ \\
\hline$\%$ Fertilized & 70 & 0 & 34 & 75 \\
\hline
\end{tabular}


$1000 \mathrm{rev} / \mathrm{min}$ for $20 \mathrm{~min}$ to pack the spermatozoa. The supernatant was decanted and discarded and each portion re-suspended in $1 \mathrm{ml}$ of one of the following media; the first (unwashed) into $8 \%$ dextran solution, the second into $\mathrm{BM}$ and the third into seminal plasma. The seminal plasma was obtained by centrifuging semen to pack the particulate and cellular material and then decanting the plasma which was pooled and frozen. The pooled plasma was thawed and centrifuged at $10,000 \mathrm{rev} / \mathrm{min}$ for $1 \mathrm{hr}$ before use.

Finding that infertility resulting from nigrosin treatment was reversible made possible an analysis of the fertility of spermatozoa after density-gradient separation. Four semen samples were treated with nigrosin and centrifuged into gradients. The spermatozoa were withdrawn, 'washed', mixed with nigrosin, layered over fresh gradients and centrifuged again. Each sample separated into two populations of spermatozoa which were isolated for insemination. A

TABLE 6

FERTILITY OF CENTRIFUGAL AND GENTRIPETAL POPULATIONS OF SPERMATOZOA

\begin{tabular}{|c|c|c|c|c|}
\hline & \multirow[b]{2}{*}{$\begin{array}{l}\text { Semen } \\
\text { samples }\end{array}$} & \multirow[b]{2}{*}{ Control } & \multicolumn{2}{|c|}{ Population } \\
\hline & & & Centripetal & Centrifugal \\
\hline$\underset{\text { inseminated }}{\text { Spermatozoa }} \times 10^{6}$ & $\begin{array}{l}1 \\
2 \\
3 \\
4\end{array}$ & $\begin{array}{c}21 \cdot 1 \\
27 \cdot 1 \\
\overline{52 \cdot 8}\end{array}$ & $\begin{array}{r}1.8 \\
5.1 \\
1.6 \\
10.8\end{array}$ & $\begin{array}{r}13 \cdot 1 \\
42 \cdot 6 \\
6.4 \\
61 \cdot 8\end{array}$ \\
\hline$\frac{\text { Blastocysts }}{\text { Corpora lutea }}$ & $\begin{array}{l}1 \\
2 \\
3 \\
4\end{array}$ & $\begin{array}{l}6 / 6 \\
7 / 7 \\
\frac{-}{3 / 6}\end{array}$ & $\begin{array}{l}1 / 6^{*} \\
0 / 8 \\
0 / 8 \\
1 / 5\end{array}$ & $\begin{array}{l}0 / 10^{*} \\
0 / 0 \dagger \\
3 / 8 \\
4 / 7\end{array}$ \\
\hline$\%$ Fertilization & & 84 & 7 & 28 \\
\hline
\end{tabular}

portion of 'washed' spermatozoa had been set aside from each sample to serve as controls. These three populations of spermatozoa from each sample (control, centripetal, and centrifugal band) were mixed with $10 \mathrm{vol}$ of BM and packed by centrifugation. They were re-suspended in seminal plasma and inseminated. The results are presented in Table 6 .

\section{DISCUSSION AND CONGLUSIONS}

Rabbit spermatozoa, centrifuged into a stabilized density-gradient medium containing Ficoll, tended to flocculate extensively (cf. Mann, 1964; Lindahl, Bodin \& Brattsand, 1963; Bedford, 1965; Metz et al., 1968). Such flocculation no doubt interfered with any attempts to separate spermatozoa according to size and/or density differences but did result in the separation of flocculated from unflocculated populations. Such flocculation was apparently associated 
with the surface charge on the spermatozoa. Spermatozoa could have interacted with each other or flocculation could have resulted from spermatozoa adhering to charged non-spermatozoal particulate matter in the semen. In any case, this observation suggests that surface charge is a common factor which could influence both sedimentation and electrophoretic separation (cf. More O'Ferrall et al., 1968), and may account for similarities in the results of the two methods for controlling sex ratio.

When flocculation was minimized by altering the spermatozoa and removing the non-spermatozoal particulate matter from the sample, a population of rapidly sedimenting spermatozoa readily separated from a population of more slowly sedimenting ones. It seemed likely that sperm activity was the basis for the separation, because heat-inactivated samples consisted only of slowly sedimenting spermatozoa. Evidence was obtained which indicated that active spermatozoa were orientated centrifugally (Plate 2). This could have resulted in active spermatozoa swimming as a population away from inactive ones (Branham, 1966).

Spermatozoa with various recognizable morphological characteristics were also not randomly distributed after centrifugation (Text-fig. 1 and Table 2). The resolution of the method for detecting such non-randomness was probably poor because the spermatozoa were free to move about between the time of stopping the centrifuge and removing the samples from the tubes ( 5 to $15 \mathrm{~min}$ ). Such autonomous movement could have been considerable. 'Flashes' from swimming spermatozoa (Branham, 1969) could be seen with $a \times 3$ hand lens in density gradients illuminated with intense oblique light. Flashing spermatozoa were observed to swim as far as $5 \mathrm{~cm}$ in $15 \mathrm{~min}$ through uncentrifuged gradients. The sampling and scoring methods no doubt also reduced the resolution. Nevertheless, the results indicated variations in the proportion of the different types at different levels in the column. This could have resulted from a direct effect of the characteristic in question on sedimentation rate or it could reflect a correlation between sperm movement and morphological characteristics.

The results of the analysis of fertilizing capacity demonstrated that fertile spermatozoa were present in both the centrifugal and centripetal populations (unless mixing occurred). The separation procedure apparently reduced the fertility of both populations but had a more pronounced effect on the slower of the two. This centripetal population generally contained only about a sixth of the number of spermatozoa in the faster populations, but the total number of spermatozoa in all samples was greater than the minimum $\left(1 \times 10^{-6}\right)$ required for maximum fertilization as reported by Wales, Martin \& O'Shea (1965). These observations suggest that the method could be useful for elucidating the characteristics of 'normal' spermatozoa by correlating morphology or activity with fertility.

The inhibitory effects of nigrosin on fertility also deserve comment. Inhibition was complete and apparently not associated with inhibition of motility. It was reversible because re-suspension in seminal plasma restored fertility. The phenomenon should be further investigated.

This procedure for separating spermatozoa into discrete populations, primarily on the basis of activity, could be useful for evaluating semen samples. 
With refinements, it should be possible to determine the proportion of active and inactive spermatozoa and perhaps even the proportion of spermatozoa with various types of progression (Branham, 1969) or specific morphological characteristics. The observed differences between semen samples were quite obvious and should be compared with existing methods for semen analysis.

These observations demonstrate that activity and flocculation influenced pre-equilibrium sedimentation rate more pronouncedly than size or density difference. Non-flocculating, inactivated spermatozoa apparently sedimented as a single population. Attempts to control genetic sex by centrifugal separation of spermatozoa should be re-investigated with this in mind.

\section{ACKNOWLEDGMENTS}

This research was supported by the United States National Institutes of Health (Child Health and Human Development post-doctoral fellowship 1 F2 HD-11, 972-01) and used equipment provided by a Ford Foundation Grant in support of Research by Dr R. A. Beatty and Dr Anne McLaren. I am greatly indebted to Dr Beatty for sponsoring this research and for his continuing interest and constant help and advice.

\section{REFERENCES}

ADAms, C. E. (1962) Artificial insemination in rodents. In: The Semen of Animals and Artificial Insemination. Tech. Commun. Commonw. Bur. Anim. Breed. Genet. 15, 316.

Beatty, R. A. (1964) Density gradient media for mammalian spermatozoa. Proc. 5th Int. Congr. Anim. Reprod. Art. Insem. Trento, 3, 276.

BEDFORD, J. M. (1965) Non-specific tail-tail agglutination of mammalian spermatozoa. Expl Cell Res. 38,654 .

Bedford, J. M. \& Bibeau, A. M. (1967) Failure of sperm sedimentation to influence the sex ratio of rabbits. F. Reprod. Fert. 14, 167.

Branham, J. M. (1966) Motility and aging of Arbacia sperm. Biol. Bull. mar. biol. Lab., Woods Hole, 131, 251.

Branham, J. M. (1969) Movements of free-swimming rabbit spermatozoa. F. Reprod. Fert. $18,97$.

Desjardins, G., Kirton, K. T. \& Hafs, H. D. (1968) Sperm output of rabbits at various ejaculation frequencies and their use in the design of experiments. F. Reprod. Fert. 15, 27.

DotT, H. M. \& WALTON, A. (1960) Effects of dilution and washing on ram spermatozoa studied by the flow dialysis technique. 7. Reprod. Fert. $1,350$.

Drevius, L. O. \& Eriksson, H. (1966) Osmotic swelling of mammalian spermatozoa. Expl Cell Res. 42, 136.

Emmens, C. W. (1948) The effect of variations in osmotic pressure and electrolyte concentration on the motility of rabbit spermatozoa at different hydrogen-ion concentrations. F. Physiol., Lond. 107, 129.

FreUnd, M. (1966) Standards for the rating of human sperm morphology. A cooperative study. Int. $\mathcal{F}$. Fert. 11, 97.

Hancock, J. L. (1952) The morphology of bull spermatozoa. F. exp. Biol. 29, 445.

Harvey, E. N. (1946) Can the sex of mammalian offspring be controlled? $\mathcal{F}$. Hered. 37, 71.

Lindahl, P. E., Bodin, N. O. \& BRATtSANd, R. (1963) Sperm agglutinating and anti-agglutinating factors in normal bull seminal plasma. Int. F. Fert. 8, 823.

LusH, J. L. (1925) The possibility of sex control by artificial insemination with centrifuged spermatozoa. F. agric. Res. 30, 893.

ManN, T. (1964) The biochemistry of semen of the male reproductive tract. Methuen, London.

Metz, C. B., Hinsch, G. W. \& ANIKA, J. L. (1968) Ultrastructure and antigens of particles from rabbit semen. F. Reprod. Fert. 17, 195.

More O'Ferrall, G. J., Meacham, T. N. \& Foreman, W. E. (1968) Attempts to separate rabbit spermatozoa by means of froth flotation and the sex ratio of offspring born. J. Reprod. Fert. 16, 243. 
Wales, R. G., Martin, L. \& O'SheA, T. (1965) Effect of dilution rate and the number of spermatozoa inseminated on the fertility of rabbits ovulated with chorionic gonadotrophin. F. Reprod. Fert. 10, 69.

WALton, A. (1924) Studies on the physiology of reproduction. I. The flocculation of sperm suspensions in relation to surface charge. Br. F. exp. Biol. 2, 13. 